\title{
Longitudinal study of Staphylococcus aureus colonization and infection in a cohort of swine veterinarians in the United States
}

\author{
Jisun Sun', My Yang ${ }^{1}$, Srinand Sreevatsan', Jeffrey B. Bender ${ }^{1}$, Randall S. Singer², Todd P. Knutson',
}

Douglas G. Marthaler ${ }^{1}$ and Peter R. Davies ${ }^{1 *}$

\begin{abstract}
Background: People working with pigs are at elevated risk of harboring methicillin resistant S. aureus (MRSA) in their nose, which is attributable to occupational exposure to animals harboring livestock adapted S. aureus. To obtain insight into the biological nature of occupationally related nasal culture positivity, we conducted a longitudinal study of 66 swine veterinarians in the USA.
\end{abstract}

Methods: The study cohort resided in 15 US states and worked predominantly with swine. Monthly for 18 months, participants self-collected nasal swabs and completed a survey to report recent exposure to pigs and other animals; the occurrence of work related injuries; and any relevant health events such as skin and soft tissue infections or confirmed staphylococcal infections. Nasal swabs were cultured using selective methods to determine the presence of MRSA and methicillin susceptible S. aureus (MSSA), and isolates were characterized by spa typing and MLST.

Results: Prevalences of S. aureus (64\%, monthly range from 58 to 82\%) and MRSA (9.5\%; monthly range from 6 to $15 \%)$ were higher than reported for the US population (30\% and 1.5\% respectively). Predominant spa types were t034 (ST398, 37\%), t002 (ST5, 17\%) and t337 (ST9/ST398 13\%), a distribution similar to that found in a concurrent study in pigs in the USA. Veterinarians were classified into three groups: Persistent carriers (PC, 52\%), Intermittent carriers (IC, 47\%) and Non-carriers (NC, 1\%). Persistent carriage of a single spa type was observed in 14 (21\%) of participants, and paired (first and last) isolates from PC subjects had minor genetic differences. Swabs from PC veterinarians carried higher numbers of S. aureus. Among IC veterinarians, culture positivity was significantly associated with recent contact with pigs.

Conclusions: Exposure to pigs did not lead to prolonged colonization in most subjects, and the higher numbers of $S$. aureus in PC subjects suggests that unknown host factors may determine the likelihood of prolonged colonization by $S$. aureus of livestock origin. Exposure to $S$. aureus and persistent colonization of swine veterinarians was common but rarely associated with S. aureus disease.

Keywords: Veterinarians, MRSA, Swine, Persistent carriage

\section{Background}

Working and living in close contact with domestic animals facilitates bidirectional interspecies transmission of microbiota. Concerns about the importance of animals as sources of antibiotic resistant pathogens have been heightened by the unveiling of healthy livestock as reservoirs of methicillin-resistant Staphylococcus aureus

\footnotetext{
* Correspondence: davie001@umn.edu

'Department of Veterinary Population Medicine, University of Minnesota, 385 ASVM, 1988 Fitch Ave, St. Paul, MN 55108, USA

Full list of author information is available at the end of the article
}

(MRSA) in many parts of the world [1-7]. While most research has focused on the ST398 lineage of livestock associated MRSA that predominates in Europe, several genotypes of $S$. aureus are adapted to livestock, and their relative prevalence varies geographically and among livestock species $[2,8-10]$.

In developed countries, approximately 20 to $30 \%$ of healthy people harbor $S$. aureus in the nasal cavity, and nasal colonization is associated with elevated risk of clinical infections [11, 12]. The most recent (2003-2004) national data for the USA estimated $28.6 \%$ and $1.5 \%$ of 
the population to harbor $S$. aureus and MRSA, respectively, in their nasal cavities [13]. S. aureus colonization is heterogeneously distributed across subsets of the population classified as 'persistent' (or permanent), 'intermittent' or 'non' carriers, although the criteria for defining persistent carriage vary [14, 15]. Bacterial, host, microbiome, and other environmental factors may influence the likelihood and duration of nasal colonization of humans with $S$. aureus [16-18], but detailed longitudinal studies of the dynamics of nasal carriage and bacterial genetic diversity are necessary to better understand this phenomenon [14]. Because some lineages of $S$. aureus are known to be host-adapted to particular avian or mammalian species $[19,20]$, and subtle genomic changes can alter host tropism [21], regular exposure of people to $S$. aureus of animal origin further complicates the poorly understood biology of nasal staphylococcal colonization.

S. aureus is considered part of the normal bacterial flora of pigs [22], and people working with live pigs are at elevated risk of being culture positive for $S$. aureus and MRSA. Notably, the predominant genotypes detected in humans with animal contact are typically those present in the animal populations with which they have contact [1, 23-29]. Because $S$. aureus are among the most numerous bacteria in bioaerosols of swine barns [30, 31], discriminating between transient contamination of superficial anatomical sites of people (e.g., upper airways or skin) and sustained colonization is problematic, particularly for workers with regular animal contact. Transient contamination may be the most common outcome in people after short term exposure to MRSA positive swine herds $[28,32,33]$. To date there have only been 2 substantial longitudinal studies of MRSA and $S$. aureus colonization in occupationally exposed swine workers, both in the Netherlands. A study of 110 farm workers sampled 6 times over a year reported that 38\% were 'persistent nasal carriers' of MRSA, but the possibility of repeated exposure and recontamination could not be eliminated [34]. A study of 137 swine veterinarians sampled 5 times over a 2 year period classified 13\% of subjects to be persistently colonized with MRSA based on consistent molecular typing of isolates [24]. The rather different estimates ( $38 \%$ vs $13 \%$ ) of persistent carriage reported in these 2 studiesmay be an artifact of the different sampling protocols and/or experimental subjects (farmers vs. veterinarians).

Fundamental questions remain about the capacity for $S$. aureus lineages disseminated from animals to colonize and cause disease in humans. Veterinarians are likely more informative subjects than farmers for elucidating long term colonization patterns following interspecies exposure as they typically are exposed to multiple herds rather than a single animal population. The goal of this study was to analyze long term patterns of $S$. aureus (including MRSA) colonization in an intensively sampled cohort of US swine veterinarians.

\section{Methods}

The specific aims of the study were to describe the frequency and duration of positive $S$. aureus and MRSA nasal cultures in a cohort of veterinarians having regular contact with varied populations of commercial swine in the USA, and to characterize the genotypes of the isolates detected. The intensity of sampling (monthly for 18 months) was designed to enable more detailed understanding of $S$. aureus colonization patterns than in previous studies of swine workers.

\section{Recruitment of study participants}

Participants for the study were recruited at the annual meeting of the American Association of Swine Veterinarians (AASV) in Denver, CO in 2012. Eligible veterinarians were members of the AASV who were US residents and typically had regular (i.e., > twice per week) professional contact with pigs. A total of 71 veterinarians provided written consent to be research subjects, of which 68 subsequently participated in sample collection. Two participants withdrew during the course of the study (one due to emigration, one due to leaving swine practice), yielding a final cohort of 66 veterinarians who completed the longitudinal sampling protocol. Participants resided in 15 US states (IA, IL, IN, MI, MN, NE, SD, TX, OK, AL, MO, PA, $\mathrm{NC}, \mathrm{MD}, \mathrm{OH}$ ), predominantly in the major swine producing regions of the Midwest and Southeast.

\section{Sample submission and survey data}

Collection materials were mailed to the participants who were given written instructions for self-collection of nasal swabs, as well as an instructional video via YouTube. Starting in July 2012, participants were contacted by monthly email and requested to collect and submit a nasal swab via mail. The email message included a link to a survey using an online tool (http://www.surveymonkey.com) for veterinarians to provide information related to recent pig contact (e.g., time since last pig contact, hours worked in the previous week, number of farms visited in the previous week), and events of physical injury and selected health events (occurrence of skin or soft tissue infections, or confirmed staphylococcal infections) occurring in the month preceding sampling. To encourage compliance, sample collection was conducted at the convenience of the participants, and follow-up emails were sent to non-responders to encourage response rates. To determine quantitative bacteriology of S. aureus, a one-time cross-sectional sampling was performed on 41 available subjects who attended the 2014 AASV meeting in Dallas, TX. 


\section{Bacteriology}

Samples were refrigerated on arrival at the University of Minnesota, and processed in 3 to 4 batches each month as samples typically were received over a 10-14 day period. For the quantitative bacteriology, all samples were collected and processed as one batch within $24 \mathrm{~h}$ of collection.

Isolation of $S$. aureus was performed using the methods described previously [35]. Nasal swabs were double enriched in Mueller-Hinton broth (BBL ${ }^{\mathrm{TM}}, \mathrm{MD}$, USA) supplemented with $\mathrm{NaCl}(6.5 \%)$ and in Phenol-Red Mannitol broth (BBL ${ }^{\mathrm{m}}$, MD, USA) supplemented with $4 \mu \mathrm{g} / \mathrm{ml}$ Oxacillin (Sigma-Aldrich, MO, USA). Broths with a color change were inoculated onto chromogenic agar plate (BBL CHROM agar MRSA, MD, USA) and Factor plate (Veterinary Diagnostic Laboratory, University of Minnesota, MN, USA) to culture MRSA and $S$. aureus, respectively. Two colonies per sample were collected for further characterization. DNA was extracted from colonies with $19.5 \mu \mathrm{l} 10 \mathrm{mM}$ Tris- $\mathrm{HCl}$ and $0.5 \mu \mathrm{l}$ Lysostaphin (both Sigma-Aldrich, MO, USA) at $37^{\circ} \mathrm{C}$ for $30 \mathrm{~min}$. PCR was used to detect mecA and perform spa typing. The primers for $m e c A$ were [F: 5' GTA GAA ATG ACT GAA CGT CCG ATA A 3', R: 5' CCA ATT CCA CAT TGT TCG GTC TAA 3'], and for spa [F: 5' AGA CGA TCC TTC GGT GAG C 3', R: 5' GCT TTT GCA ATG TCA TTT ACT G 3']. PCR master mix (USB HotStart-IT FideliTaq, affymetrix, CA, USA) was used to amplify the DNA under the following conditions: $95^{\circ} \mathrm{C}$ for 2 min, $94{ }^{\circ} \mathrm{C}$ for 30 s, $55{ }^{\circ} \mathrm{C}$ for 30 s, $72{ }^{\circ} \mathrm{C}$ for 1 min with 30 cycles and $72{ }^{\circ} \mathrm{C}$ for $10 \mathrm{~min}$. All PCR products were visualized in $1 \%$ agarose gel with SYBR Safe dye in $1 \mathrm{X}$ TAE buffer (Tris-Acetate-EDTA, Thermo Fisher Scientific Inc., MA USA) for $40 \mathrm{~min}$ at $200 \mathrm{~V}$.

\section{Quantitative bacteriology}

Nasal swabs collected from the 41 subjects were transported in one batch on ice to the laboratory and placed in $1 \mathrm{ml}$ Mueller-Hinton broth tubes within $24 \mathrm{~h}$. Tenfold dilutions were prepared from 100ul broth (up to 10 $\left.{ }^{-4}\right)$ and 100ul from each dilution was spread on a Factor plate and incubated at $37{ }^{\circ} \mathrm{C}$ for $22 \mathrm{~h}$. before counting by observers blinded to the carrier status of subjects to determine $\mathrm{CFU} / \mathrm{swab}$.

\section{Molecular typing and analysis}

All selected $S$. aureus isolates were subtyped using spa typing [36]. After amplification of spa, PCR products were cleaned up with Illustra Exoprostar, (GE Healthcare Bio-sciences, PA, USA) and sequenced at the University of Minnesota Genomics Center. Sequences aligned using Sequencher 5.1 software (Gene Codes Corporation, MI, USA) were submitted to the Ridom spa typing database (http://spa.ridom.de/index.shtml).

Multi-locus sequence typing (MLST) of S. aureus was performed following methods reported previously via the MLST database of $S$. aureus (http://saureus.mlst.net) [37]. MLST typing was performed purposively so that at least one isolate from each spa type detected was also evaluated by MLST.

\section{Definition of carrier status}

Consistent with previous studies [14, 15, 24], we classified subjects by carrier status to be non-carriers (NC), intermittent carriers (IC), or persistent carriers (PC). A carrier index (range 0 to 1 ) was defined as the proportion of sampling events that yielded a $S$. aureus (including MRSA) isolate. Non-carriers were defined as subjects that were never positive for S. aureus (including MRSA), and intermittent carriers were culture positive at least once occasion with a carrier index of $<0.8$. Persistent carriers had a carrier index of 0.8 or greater. The cut-off of 0.8 was based on post hoc evaluation of the frequency distribution of the carrier index and was considered conservative (i.e., biased against false positive misclassification of persistent carriage). PC subjects were further classified as true persistent carriers (TPC) if a single spa type of $S$. aureus was recovered at all positive sampling events.

\section{PCR testing for scn, chp, sak for the immune evasion cluster (IEC)}

A purposively selected subset of 116 isolates was tested for presence of scn, chp, and sak of the IEC, considered as markers of adaptation to humans. The selection protocol utilized 4 categories of veterinarians 1) TPC subjects colonized with 1 spa type; 2) PC subjects colonized with more than 1 spa type; 3) IC subjects; 4) IC subjects colonized at least once with MRSA. For TPC subjects (category 1), the first and last isolates obtained were selected for each veterinarian. For category 2 subjects in whom the detected genotype changed over time, 2 isolates from each spa type were chosen up to 4 isolates per veterinarian (i.e., 2 predominant spa types). For category 3 subjects, only the predominant spa type was selected. Both MRSA and MSSA were detected from 4 IC subjects during the study period (category 4), and one MRSA isolate and an isolate of the predominant MSSA spa type were selected. Thus, a total of 24 MRSA and 92 MSSA were tested for scn, chp, sak following the methods previously described [38]. Annealing temperatures for the scn, chp and sak were $63{ }^{\circ} \mathrm{C}, 51.5{ }^{\circ} \mathrm{C}$ and $53{ }^{\circ} \mathrm{C}$, respectively. All PCR products were electrophoresed in $1 \%$ agarose gel stained with SYBR Safe dye in 1X TAE buffer (Tris-Acetate-EDTA, Thermo Fisher Scientific Inc., MA USA) for $40 \mathrm{~min}$ at $200 \mathrm{~V}$ and visualized on a UV transilluminator. ATCC 700698 (Mu3), ATCC 700699 (Mu50) and ATCC 25904 (Newman) were used as positive controls for scn, chp and sak, respectively. 


\section{Whole genome sequencing}

Eighteen isolates were selected from 9 persistent carriers (three subjects per sequence type) to evaluate genomic variation across the study period. Isolates from the first (month 1) and last (mostly month 18) samples collected for each subject were included. One veterinarian was colonized with ST5-t062 for 11 months, then ST398t011 S. aureus for the remaining 7 months. For this subject, ST5-t062 isolates from month1 and month11 were selected. Another veterinarian was colonized with ST398-t034 MRSA followed by a closely related spa type (ST398-t011 MRSA) for the final 4 months. Genomic DNA was extracted from overnight cultures in LB (Lysogeny broth, BD Difco ${ }^{\mathrm{Tm}}$, NJ, USA) using the Qiagen Blood and Tissue Kit (Valencia, CA, USA) following the manufacturer's instructions. Approximately $10 \mathrm{ng}$ of extracted DNA per sample was sent to University of Minnesota Genomics Center (Minneapolis, MN, USA). Independent next generation sequence (NGS) libraries (Nextera DNA Library Preparation Kit, CA, USA) were created for each sample, pooled onto a single lane HiSeq 2500 rapid-run, and 250 bp paired-end reads were generated. Runs yielded an average of 2.3 million reads per sample, and $82 \%$ of reads had a quality score (Phred +33 ) greater than 30 (details described in Additional file 1). To estimate genetic distances, the isolates were aligned using Mauve (ver.2) and single nucleotide polymorphisms (SNPs) from aligned genomes were extracted and imported into MEGA 7.0 software to generate a maximum likelihood phylogenetic tree employing 100 bootstrap iterations.

\section{Comparison with S. aureus isolates from veterinarians and from pigs in the USA}

The prevalence and genotypic characterization of a geographically diverse sample of $S$. aureus collected from pigs in the USA was recently published [8]. The majority (36 of 38) of farms included in that study were served by 36 veterinary participants of the current study, providing a congruent time-space window to underpin the comparison of genotypes detected in US pigs and swine veterinarians. Spa types from the current study and from the study of pigs were categorized to be 'shared' (if detected in both species), 'swine only' or 'human only'. A minimal spanning tree (MST) for clustering of spa types was constructed using the Bionumerics 7.1 (Applied Maths, SintMartens-Latem, Belgium).

\section{Statistical analysis}

Univariate analysis was performed to evaluate associations between culture positivity of $S$. aureus and working activities related with occupational exposure using $\mathrm{R}$ studio (Version 0.99.892). The exposures related to animal contact were: last contact with pigs (categorical variable, 0 : same day; 1: previous day, 2: 2 days previously, 3: 3 days, 4: more than 3 days); hours of pig contact (continuous); and the number of farms visited in the previous week (continuous). As these self-reported exposure variables were correlated, multivariable analysis was limited to last contact with pigs, which was considered the most reliable and relevant variable with respect to transient contamination. To account for repeated observations on the same subjects over time, a two-way nested mixed-effects model was used, with samples nested within veterinarians, and the fixed effect of time since last contact with pigs (Days: same day vs. more than 1 day). The model was performed in $\mathrm{R}$ software environment 3.0 , via lme4 package as follows:

$$
\operatorname{Logit}\left(\mathrm{p}_{\mathrm{i}}\right)=\text { Intercept }+ \text { Days }_{\mathrm{i}}+\text { Vet }_{\mathrm{i}}+\text { Sample }_{\mathrm{ij}}
$$

In addition, wearing a facemask at last pig contact, and occurrence of injuries by livestock or soft tissue infections were also evaluated. The numbers $\log _{10} \mathrm{CFU}$ (colony forming unit) of putative S. aureus colonies per swab was compared between intermittent carriers and persistent carriers using Mann-Whitney U test.

\section{Results}

Two veterinarians withdrew from the study due to emigration or altered work circumstances, leaving a cohort of 66 swine veterinarians who provided monthly nasal swabs. One veterinarian stopped working with swine after 7 months, but completed the sampling protocol and was retained in the study. Compliance with swab submission and survey completion was over 99\% for both nasal swab submission (1179/1188) and survey submission (1177/1188). The median interval between sample collection and sample processing was 4 days (IQ range: 3-5 days; range: 1-35 days) and was not associated with the likelihood of culture positivity $(p=0.99)$.

Overall, S. aureus was detected in 63.7\% (757/1188) of monthly nasal swab samples (yielding 1356 S. aureus isolates characterized) and MRSA in 9.5\% (113/1188) samples (yielding 213 MRSA isolates characterized). The monthly apparent prevalence of $S$. aureus ranged from $58 \%$ to $82 \%$, while apparent prevalence of MRSA ranged from 6 to 15\%, and there was no indication of seasonal or longer term trends in prevalence over the course of the study (Fig. 1). MRSA was detected at least once during the study in 18 (27\%) of subjects.

At the individual level, the proportion of positive sampling events ranged from $0 \%$ (one veterinarian) to $100 \%$ (18 veterinarians) and was clearly bimodally distributed (Fig. 2). In univariate analyses, the likelihood of a culture positive result was negatively associated with the interval between the last contact with pigs and collection of the sample $(P=0.001)$, and positively associated with the 


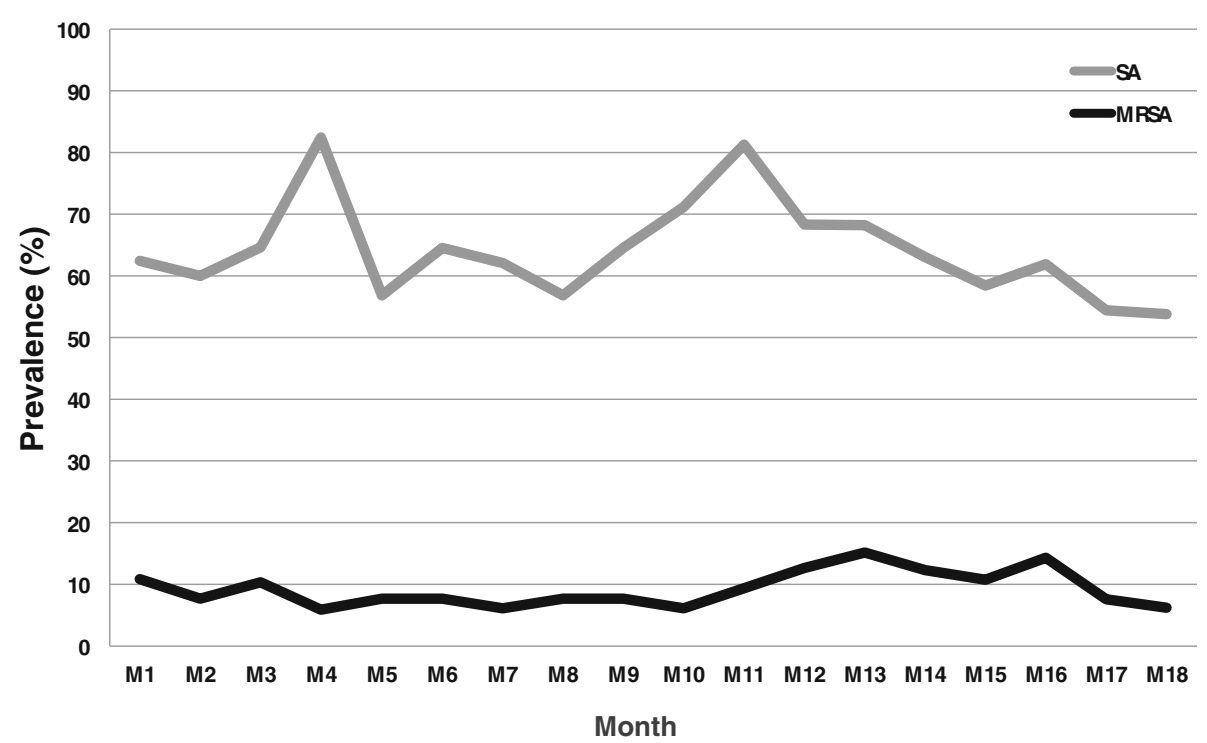

Fig. 1 Proportion of S. aureus and MRSA positive nasal swabs from a cohort of swine veterinarians sampled monthly from July 2012 to December 2013

hours of pig contact per week $(P=0.02)$, but not associated with the number of farms visited $(P=0.09)$ in the previous week. Multivariable analysis to adjust for repeated observations on the same veterinarians showed the odds or a positive culture were reduced by $33 \%$ for samples collected one day or longer after pig contact $(\mathrm{OR}=0.67 ; 95 \%$ CI: 0.46-0.96) compared with same day collection. Three subjects reported having a staphylococcal infection during the study (2 MSSA, 1 MRSA), all of which were described as localized and did not lead to hospitalization or time off work. This corresponds with an incidence of 2.5 cases per 1000 person months.
Of the 66 subjects, 31 (47\%) were classified as intermittent carriers and 34 (52\%) were classified as persistent carriers of $S$. aureus. Based on consistent detection of a specific spa type at all culture positive samplings, 14 (21\%) veterinarians were classified as true persistent carriers (TPC). The TPC group included the veterinarian who ceased working with pigs after 7 months but remained positive with ST398/t034 (methicillin susceptible) for the remaining 10 months. The majority $(60 \%)$ of MRSA isolations were from 4 PC subjects who were positive for MRSA (all ST398) on at least 15 occasions. One of the PC MRSA subjects, who consistently

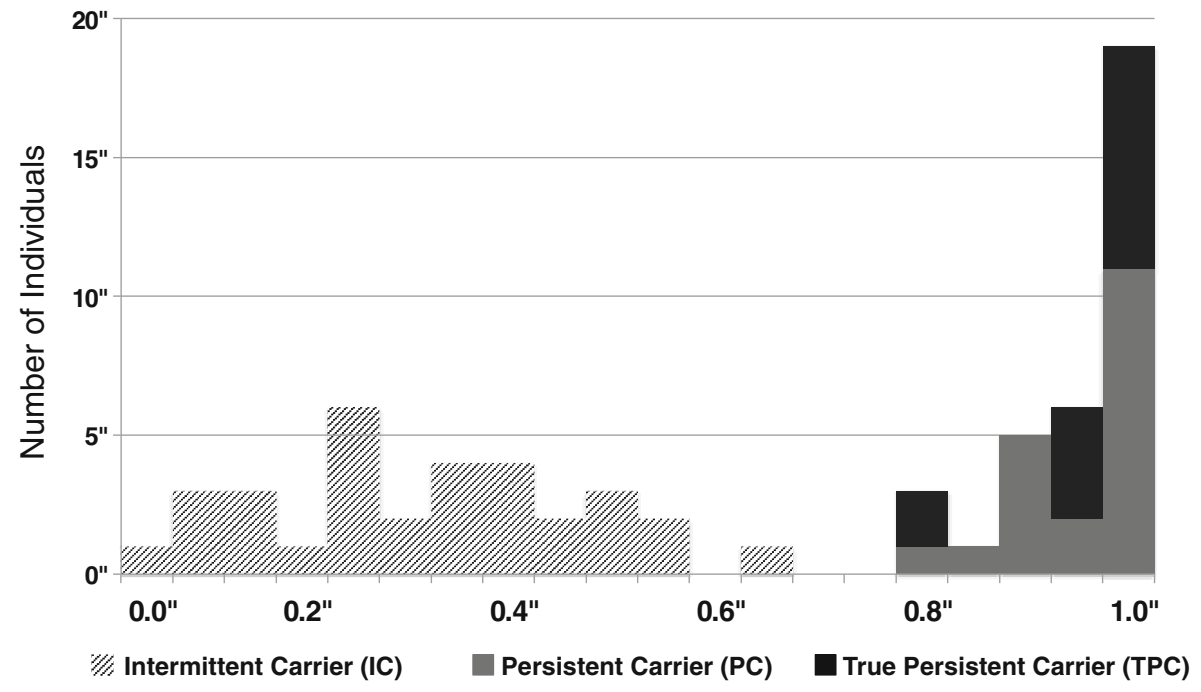

Fig. 2 Histogram of the proportion of sampling events yielding a S. aureus isolate ("Carrier index") 
harbored ST398/t034 MRSA, worked exclusively in one production system where this spa type occurs at high prevalence, and which was used as a MRSA positive control farm in the related swine study [8].

\section{Whole genome sequencing}

Comparison of the isolates from 9PC subjects (3 each of sequence types ST398, ST9 and ST5) generally indicated close genetic similarity between the first and last isolates collected. All paired isolates within subjects had 0.01 and 0.2 nucleotide differences per site within ST5 and ST398 respectively (Fig. 3). Pairs of isolates of the same genotype (ST9-t337) showed greater similarity within subjects than between subjects (Fig. 3b).

Overall, the $S$. aureus isolates were distributed among 27 spa types within 8 MLST sequence types (Table 1). Three sequence types (ST398, ST5, ST9) constituted over $94 \%$ of the $S$. aureus isolates. Over the course of

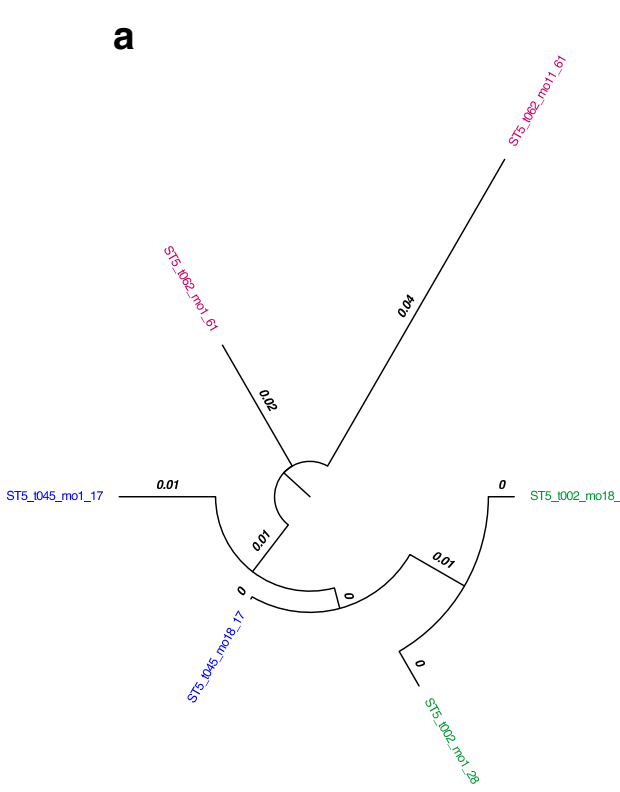

C

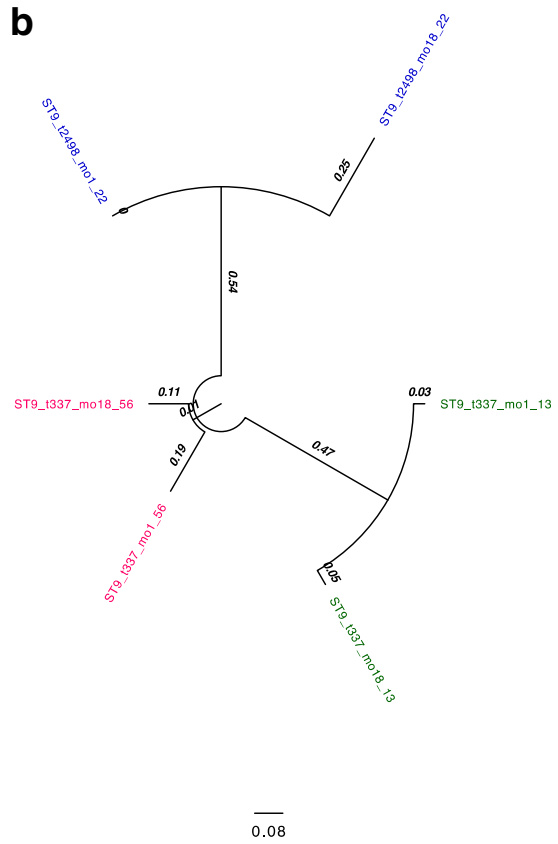

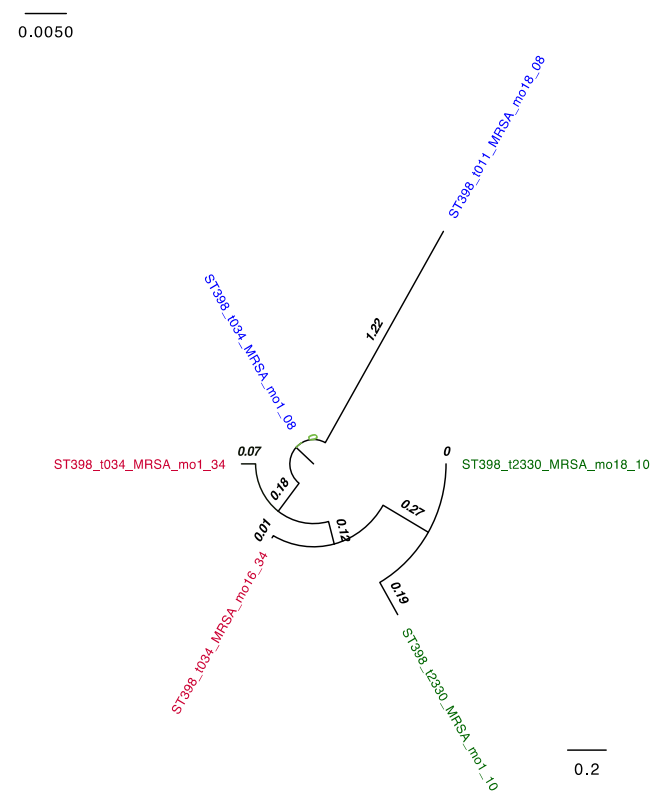

Fig. 3 Genomic distance between isolates from beginning of study and the end of study in persistent carriers displayed by Sequence Type (a) ST5, b) ST9, c) ST398). Within sequence type, the isolates from same person were colored with same color. Names of each isolate were described by ST_spa type_ (if MRSA)_sampling month_vetID. The bar scales on each ST indicate number of nucleotide difference per site 
Table 1 Numbers (\%) of spa types of S. aureus and MRSA isolated from swine veterinarians, by MLST type

\begin{tabular}{|c|c|c|c|}
\hline Sequence type & Spa type & S. aureus $(n=1356)$ & $\operatorname{MRSA}(n=213)$ \\
\hline \multirow[t]{11}{*}{ ST398 } & t034 & $462(34.1)$ & $116(54.5)$ \\
\hline & t571 & $71(5.2)$ & 0 \\
\hline & t011 & $63(4.6)$ & $12(5.6)$ \\
\hline & $\mathrm{t} 337^{\mathrm{a}}$ & $20(1.5)$ & 0 \\
\hline & $\mathrm{t} 3446^{\mathrm{a}}$ & $19(1.4)$ & 0 \\
\hline & $\mathrm{t} 1250$ & $8(0.6)$ & 0 \\
\hline & t2330 & $6(0.4)$ & $44(20.7)$ \\
\hline & t2876 & $24(1.8)$ & 0 \\
\hline & t7160 & $13(1.0)$ & 0 \\
\hline & $\mathrm{t} 1255$ & $3(0.2)$ & 0 \\
\hline & & $650(51.8)$ & $172(80.8)$ \\
\hline \multirow[t]{7}{*}{ ST5 } & t002 & $238(17.6)$ & $27(12.7)$ \\
\hline & t045 & $69(5.1)$ & 0 \\
\hline & t062 & $19(1.4)$ & 0 \\
\hline & t242 & 0 & $3(1.4)$ \\
\hline & t570 & $12(0.9)$ & 0 \\
\hline & t856 & $3(0.2)$ & 0 \\
\hline & & $341(25.1)$ & $30(14.1)$ \\
\hline \multirow[t]{7}{*}{ ST9 } & $\mathrm{t} 337^{\mathrm{a}}$ & $178(13.1)$ & 0 \\
\hline & t2498 & $47(3.5)$ & 0 \\
\hline & t10494 & $11(0.8)$ & 0 \\
\hline & $\mathrm{t} 3446^{\mathrm{a}}$ & $9(0.7)$ & 0 \\
\hline & t1334 & $3(0.2)$ & 0 \\
\hline & $\mathrm{t} 1430$ & $2(0.1)$ & 0 \\
\hline & & $289(18.4)$ & 0 \\
\hline \multirow[t]{2}{*}{ ST8 } & t008 & 0 & $11(5.1)$ \\
\hline & t2196 & $18(1.3)$ & 0 \\
\hline \multirow[t]{2}{*}{ ST30 } & t338 & $4(0.3)$ & 0 \\
\hline & t363 & $1(0.1)$ & 0 \\
\hline ST72 & $\mathrm{t} 126$ & $30(2.2)$ & 0 \\
\hline ST278 & t330 & $22(1.6)$ & 0 \\
\hline ST2007 & t8314 & $1(0.1)$ & 0 \\
\hline
\end{tabular}

${ }^{\mathrm{a}}$ Two sequence types were identified within $\mathrm{t} 337$ and $\mathrm{t} 3446$ isolates

the study, ST398 isolates were detected at least once in $63(83 \%)$ veterinarians; ST5 isolates in 43 (65\%) veterinarians, ST9 in 29 (56\%) veterinarians; and other MLST types in $8(12 \%)$ veterinarians. The 3 predominant MLST types (ST398, ST5, ST9) were all isolated at least once from $23(35 \%)$ subjects. Within each of these 3 MLST types, a single spa type (t034, t002, and $\mathrm{t} 337$, respectively) constituted approximately $70 \%$ of isolates. Two of these sequence types, ST398 (80.8\%) and ST5 (14.1\%), accounted for almost $95 \%$ of all MRSA isolates, with remainder being ST8/t008, a common human MRSA variant considered unlikely to be of swine origin.
The detection patterns of specific spa types over time varied enormously from highly consistent presence of individual spa types in TPC subjects, to very inconsistent patterns with multiple spa types detected in individual veterinarians over time (Fig. 4a, b). More than one spa type was detected in 71 (6\%) of the monthly nasal swab samples. It is also notable, that in some of the PC and IC subjects a single spa type was detected over multiple consecutive months, but thereafter other spa types were detected.

In the quantitative comparison of $\mathrm{PC}$ and IC veterinarians among 41 AASV members sampled in 2014, PC veterinarians (18 of $22 ; 82 \%$ ) were more likely to be nasal culture positive than IC (4 of 19, 21\%). Culture positive swabs from PC subjects harbored approximately 2 logs more $S$. aureus per swab than positive swabs from IC subjects $(P=0.05)$. Spa typing of isolates from this non-selective procedure yielded the same spa types found at other months for each TPC subject, and the same spa type detected in the most recent positive culture for 2 PC subjects.

\section{IEC genes testing}

Approximately 10\% (one MRSA and 11 MSSA) of the 116 tested isolates were positive for two or three of the IEC genes (Table 2). A single isolate (t2196, ST8) was only positive for the $s c n$ and $s a k$ genes. The majority of isolates positive for IEC genes were spa types likely to be of human origin apart from the t011 (ST398), t5883 (ST398) and t002 (ST5) isolates.

\section{Comparison with S. aureus isolates from veterinarians and from pigs in the USA}

Spa types from the current study and a previous pig study [8] were categorized as 'shared' if detected in both species, 'swine only' or 'human only'. Thirteen spa types were shared and accounted for $83 \%$ and $92 \%$ of all isolates from veterinarians and pigs, respectively. Twenty spa types were found only among swine isolates while 14 spa types were identified only among veterinary isolates (Table 3). A minimal spanning tree (MST) analysis for clustering of spa types from the swine and veterinary studies was constructed (Fig. 5). As expected, isolates were clustered together by sequence type. ST9 isolates were more likely to be found among pig isolates while spa types belonging to ST398 and ST5 were relatively more frequent among isolates from veterinarians.

\section{Discussion}

The high prevalence of $S$. aureus recovered from nasal cultures reflects the increased exposure to $S$. aureus occurring in livestock environments [4, 26, 39]. Although some influence of methodological differences cannot be eliminated, the overall $S$. aureus prevalence (64\%) in swine veterinarians is approximately double that estimated in 


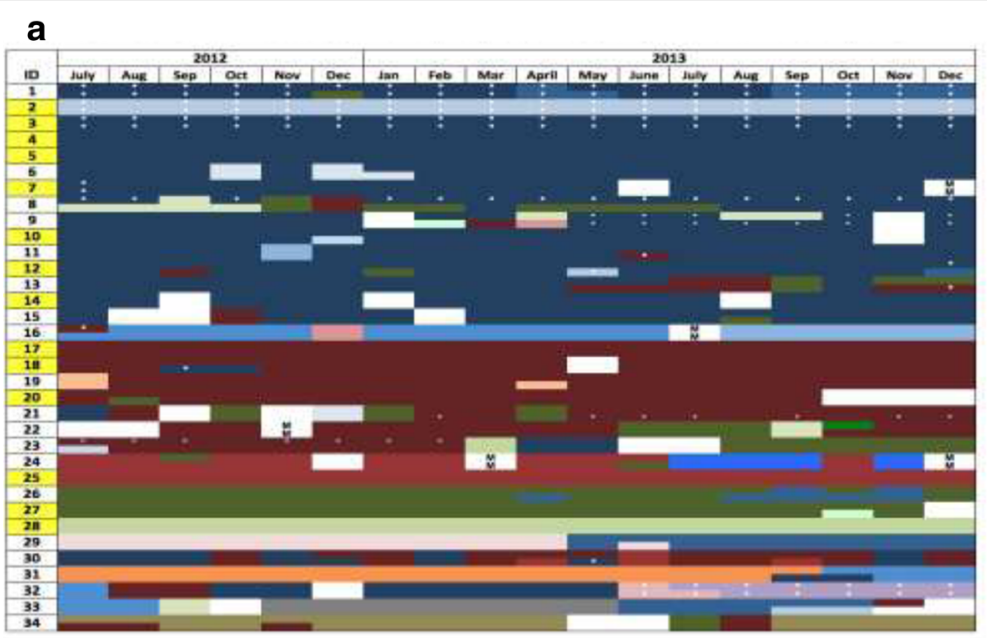

b

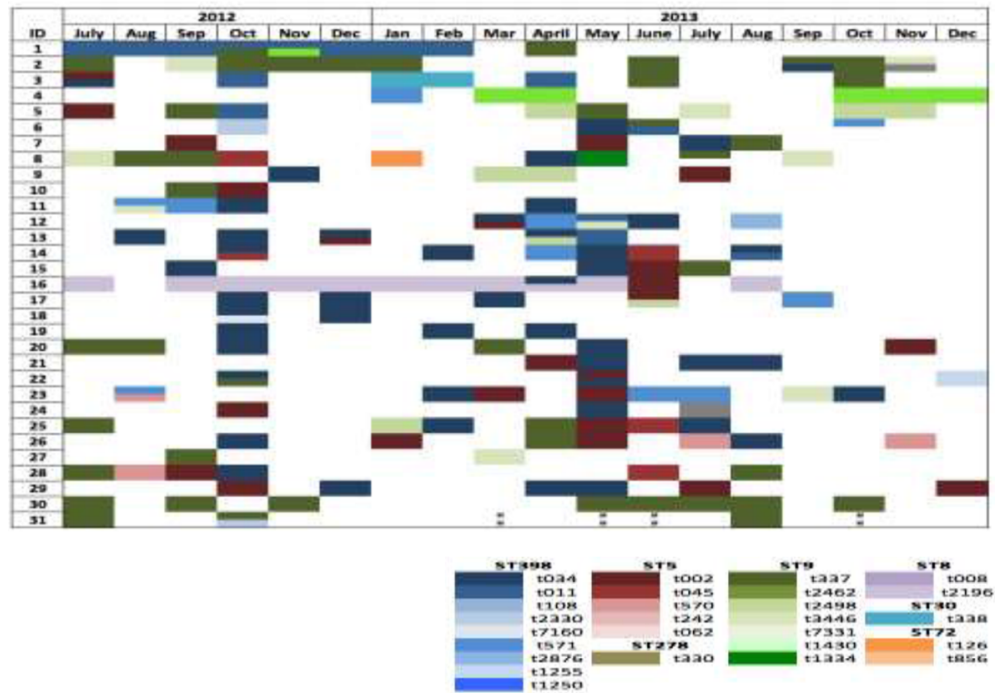

Fig. 4 Patterns of detection of S. aureus spa types in veterinarians categorized as permanent carriers (a) and intermittent carriers (b). Missing samples are indicated as ' $\mathrm{M}$ ', white spaces indicate culture negative events; white dots signify methicillin resistant isolates (typically 2 isolates typed per month). Yellow boxes in (a) indicate true persistent carriers. Colors also reflect the MSLT type of the major sequence types being ST398 (blue shades), ST5 (red shades), and ST9 (green shades)

studies of the overall US population [13] and in other developed countries $[11,12]$. However, it is very similar to a $72 \%$ prevalence in a study of Dutch swine veterinarians [24]. The prevalence of MRSA (9.5\%) was also higher than reported in the US population (1.5\%), but was substantially lower than in the Dutch swine veterinarians (44\%), which likely reflects the lower prevalence of MRSA in the US swine industry relative to the Netherlands $[4,8,40]$.

A substantial majority $(>84 \%)$ of $S$. aureus isolates in this study were deemed likely to be of swine origin based on several criteria. A parallel study of 38 US swine farms, sampled by a subset of the veterinarians in the current study, found that three MLST sequence types (ST9, ST398, ST5) constituted over 99\% of swine isolates, with spa types $\mathrm{t} 337$, t034, and t002 predominating [8]. The same sequence types and spa types were similarly predominant among the veterinarians, generally lacked the IEC genes, and were predominantly (85\%) tetracycline resistant [41]. The absence of the IEC genes, together with tetracycline resistance, has been used previously to differentiate isolates of human and animal origin $[42,43]$ and our data suggest that $S$. aureus acquired from swine may largely displace $S$. aureus of human origin in the nasal flora of swine veterinarians. However, given that more than one spa type was often detected in individual samples, and only 2 isolates per sample were categorized, it is possible that carriage of relatively low numbers of human $S$. aureus by veterinarians went 
Table 2 S. aureus isolates testing positive for IEC

\begin{tabular}{llllllll}
\hline & Month $^{a}$ & ID & MRSA & spa type (ST) & scn & sak & chp \\
\hline 1 & 04 & 24 & MSSA & t126 (ST72) & + & + & + \\
2 & 04 & 57 & MSSA & t330 (ST278) & + & + & + \\
3 & 04 & 61 & MSSA & t062 (ST5) & + & + & + \\
4 & 06 & 19 & MSSA & t5883 (ST398) & + & + & + \\
5 & 07 & 05 & MSSA & t338 (ST30) & + & + & + \\
6 & 09 & 44 & MSSA & t2196 (ST8) & + & + & - \\
7 & 12 & 61 & MSSA & t062 (ST5) & + & + & + \\
8 & 12 & 61 & MSSA & t011 (ST398) & + & + & + \\
9 & 16 & 57 & MSSA & t330 (ST278) & + & + & + \\
10 & 17 & 41 & MRSA & t008 (ST8) & + & + & + \\
11 & 17 & 66 & MSSA & t002 (ST5) & + & + & + \\
12 & 18 & 66 & MSSA & t002 (ST5) & + & + & + \\
\hline
\end{tabular}

${ }^{\mathrm{a} M o n t h}$ of sampling from month 1 to month 18

undetected [44], and that animal contact added to, rather than displaced human $S$. aureus. Regardless, the data suggest that animal exposure alters the composition of the nasal $S$. aureus populations of swine workers. Any resultant impact on risk of clinical infection will depend

Table 3 Spa type comparison between swine and veterinary isolates

\begin{tabular}{lll}
\hline Shared & Swine only & Human only \\
\hline t002 & t899 & t008 \\
t011 & t5883 & t045 \\
t034 & t5838 & t062 \\
t10494 & t5462 & t1250 \\
t1255 & t3232 & t126 \\
t1334 & t306 & t1430 \\
t242 & t2582 & t2196 \\
t2498 & t2462 & t2330 \\
t337 & t2315 & t2876 \\
t3446 & t1793 & t330 \\
t570 & t14851 & t338 \\
t571 & t1419 & t363 \\
t8314 & t11744 & t856 \\
& t11374 & t922 \\
& t11241 & \\
& unknown1 & \\
& unknown2 & \\
& unknown3 & \\
& unknown5 & \\
& & \\
& &
\end{tabular}

*Repeat succession of unknown types: Unknown1 (r07r16r23r23r02r12r17r23r 02r34), Unknown2 (r07r16r16r16r23r23r02r12r23r02r34), Unknown3 (r07r16r1 6r23r02r12r23r02r34), Unknown4 (r08r475r2r25r2r25r34r34r25),

Unknown5 (r07r16r23r23r02r23r02r34) on the relative persistence, transmissibility, and pathogenicity of $S$. aureus of swine origin compared with human adapted variants.

The primary goal of this study was to understand the persistence of $S$. aureus of swine origin in occupational groups that are in close contact with pigs. Several previous studies of livestock workers have examined this question, with varying outcomes [3-5, 26, 29, 32, 39]. Most studies focused on MRSA alone, and the frequency and duration of sampling has varied widely. A common obstacle to inference has been the inability to differentiate repeated contamination of the nasal mucosa from true persistent colonization [45], particularly for farmers who are repeatedly exposed to the same herd (and $S$. aureus populations) [39]. We specifically studied veterinarians because they generally visit multiple farms and therefore should be exposed to more diverse $S$. aureus populations.

Longitudinal studies of nasal carriage of $S$. aureus in humans typically classify subjects as persistent carriers, intermittent carriers, and non-carriers $[14,46]$. It is believed that a subset (usually of the order of 20\%) of healthy people are persistently colonized with $S$. aureus $[15,47]$, and this is associated with higher risk of $S$. aureus clinical infections [48, 49]. Both host genetic factors and microbial factors may play a role in determining duration of carriage [16], and it was recently reported that presence of $S$. lugdunensis in the nose may suppress S. aureus populations [18]. Currently, there is no accepted consensus for defining persistent carriers, and categorization of individuals will be influenced by study design (particularly the frequency and duration of sampling), and the cut-off (carrier index) used to define carriage status [46]. We employed a post-hoc epidemiological approach to establish a cut-off (carriage index $>0.8$ ) to define persistent carriage. The same criterion has been employed in previous studies [14, 46]. The bimodal distribution of the frequency of culture positive samples among veterinarians (Fig. 2) suggests that even in environments with high exposure to S. aureus of animal origin, individual host characteristics are likely important determinants of the persistence of colonization. This inference is further supported by the substantially higher numbers of $S$. aureus recovered from nasal swabs from PC compared with IC veterinarians, which is consistent with quantitative studies performed in humans both with [39] and without [50] known livestock association.

The proportion of persistent carriers $(52 \%, 34 / 66)$ was considerably higher, and the proportion of non-carriers $(<2 \%, 1 / 66)$ considerably lower, than reported in people without livestock contact $[14,46,51]$. This may reflect unusually frequent exposure to $S$. aureus that can occur in intensive swine facilities [52]. Very similar rates of apparent persistent carriage of $S$. aureus were also 


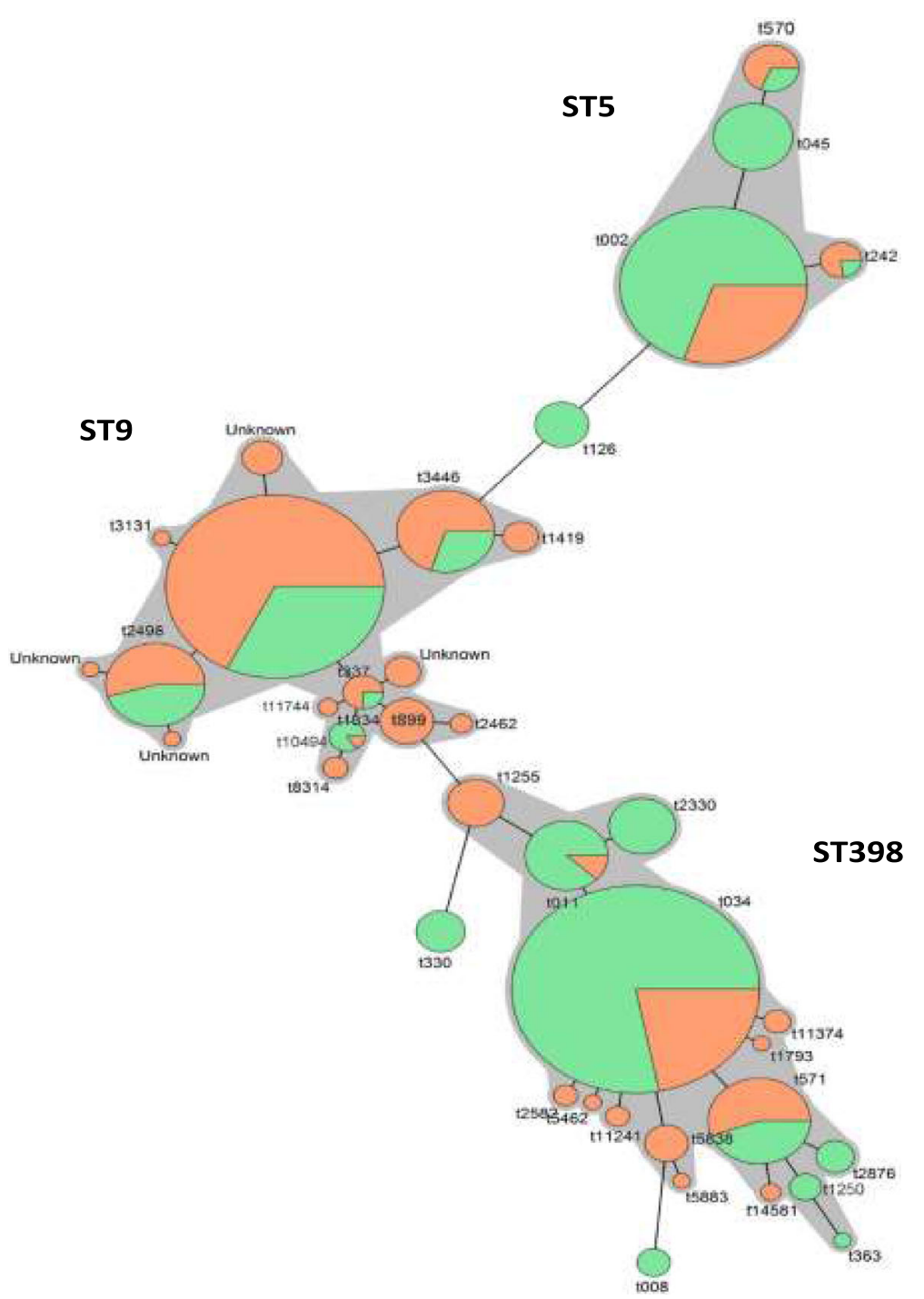

Fig. 5 Genetic relatedness of S. aureus isolates from swine $(n=1193)$ and veterinarians $(n=1659)$. Each node in this minimum spanning tree depicts one of 38 spa types identified from swine and swine veterinarians. The size of circles denotes the number of isolates. Swine isolates and vet isolates are color coded with orange and green, respectively

observed in recent longitudinal studies of swine farmers (52\%, [39]) and swine veterinarians (47\%, [24]) in the Netherlands, although the sampling protocols and criteria for defining persistence in those studies differed.

The associations observed between culture positivity and time since last pig contact and hours of pig contact in the prior week are consistent with previous studies indicating that occupational exposures to animals often result in transient contamination [28, 32, 53]. Collection of swabs on the same day following pig contact substantially increases the odds of a positive culture, therefore sampling schedules will influence estimates of prevalence. The variability of spa types detected over time in the IC group (Fig. 4b) also attests to multiple exposures of swine veterinarians to diverse $S$. aureus populations.
Although early research of persistent carriage in humans suggested that people were colonized over the long term by a single $S$. aureus variant, more recent studies indicate that $S$. aureus variants are often replaced over time [14, 46, 51]. Ritchie (2015) sampled 122 healthy young adults weekly over 13 weeks and described 3 patterns of persistent carriage, being continuous carriage of a single spa type; an abrupt change from one spa type to another; and periods of co-carriage with two spa types. Although 'strain turnover' was observed in both intermittent and persistent carriers in that study, the majority (63\%) of PC subjects were colonized by a single spa type. We made similar observations in the veterinary cohort where all 3 patterns of PC were observed, and a substantial proportion (41\%; 14 of $34 \mathrm{PC})$ of the $\mathrm{PC}$ 
veterinarians were classified as 'true persistent colonization' due to the repeated presence of a single spa type over 18 months. This slightly lower proportion (41\% vs. $63 \%$ ) of TPC could be an artifact of the longer sampling period (18 months vs. 13 weeks) providing more opportunity for strain turnover, or the additional exposure to $S$. aureus variants that occurs in the livestock environment. Our observations with WGS provided further evidence that true persistent colonization with animal origin of $S$. aureus can occur in some subjects. The paired isolates from each persistent carrier (18 months apart) had shorter genomic distances than seen between isolates of same spa type but from different individuals. A similar inference was reached in a previous study using whole genome mapping of isolates from 16 Dutch veterinarians [54]. Although it is not possible to eliminate the possibility of repeated reacquisition of the same variant from pigs by veterinarians, this is considered unlikely over an extended period unless they were exposed to homogeneous swine populations harboring few $S$. aureus variants. However, repeated reacquisition from pigs can be eliminated for one subject in our study who remained culture positive for ST398t034 MSSA for 9 months after leaving swine practice. Current evidence suggests that a substantial proportion of swine veterinarians become colonized with $S$. aureus of swine origin for at least 18 months to 2 years [24], and can harbor substantial numbers of these organisms. Furthermore, although several studies indicate that ST398 MRSA are less transmissible among people than MRSA of human origin, transmission from veterinarians to their families, who also may become persistently infected, has been clearly demonstrated $[54,55]$. Also, persistent colonization of humans with swine $S$. aureus isolates may be a mechanism for transmission among herds. This is arguably inconsequential given that $S$. aureus is part of the normal commensal flora of swine, but could be problematic for efforts to prevent MRSA transmission among herds [56].

The fact that some swine workers harbor substantial numbers of $S$. aureus with genotypes consistent with swine origin has implications regarding occupational health. However, the human health consequences of livestock associated S. aureus are not well defined, and available information is largely limited to ST398 MRSA. In the USA, to date $S$. aureus of animal origin appear to have had negligible impact on human health. The reported incidence of S. aureus infections in this study (2.5 per 1000 person months, or $3 \%$ per veterinary-year) is similar to that reported in a study of Iowa residents ( 2.7 per 1000 person months). Furthermore, spa types linked to livestock represented only $1 \%$ of $S$. aureus isolates, and $0.24 \%$ of MRSA isolates, from human clinical infections in the pig dense state of Iowa [57]. In contrast, a substantial study in Denmark showed increased likelihood of infection with specific livestock associated MRSA, but not increased MRSA infection risk overall, in people living in pig dense areas [58]. Transmission from swine workers into the broader community is a plausible explanation. However, despite the substantial exposure to S. aureus of swine origin that occurs in intensive production environments, there are remarkably few reports of medically significant infections occurring in swine workers $[45,59]$. To date, we are unaware of any studies demonstrating increased risk of clinical $S$. aureus infections in livestock workers. A recent prospective study of swine farm workers in Holland found that carriage of ST398 MRSA was not associated with elevated risk of infections, healthcare contact, or measures of reduced quality of life [60]. Somewhat surprisingly, the small numbers of fatal human cases of ST398 infection have occurred in people without known livestock contact, but who were generally medically compromised [61]. Even though there are a small number of fatal human cases of ST398 infections, it is important to better understand the interchange of $S$. aureus between humans and animals and the implications for the transfer of resistance elements.

\section{Conclusions}

Swine veterinarians are continually exposed to S. aureus of swine origin when working with pigs. This longitudinal study confirmed that the outcomes of this occupational exposure range from short term contamination of the nasal cavities (reflected in higher prevalence of detection in samples collected soon after animal exposure) to apparent long term colonization (at least 18 months) in some individuals in whom the same spa type was detected repeatedly throughout the study. Exposure to pigs did not lead to prolonged colonization in most subjects, and the higher numbers of $S$. aureus in PC subjects suggests that unknown host factors may determine the likelihood of prolonged colonization by $S$. aureus of livestock origin. Although exposure to $S$. aureus and persistent colonization of swine veterinarians was common, the few reports of clinical $S$. aureus infections reported were of minimal medical significance.

\section{Additional file}

Additional file 1: Supplement methods for whole genome sequencing analysis. (DOCX $108 \mathrm{~kb}$ )

\section{Abbreviations}

AASV: American Association of Swine Veterinarians; AL: Alabama; ATCC: American Type Culture Collection; CFU: Colony forming unit;

CO: Colorado; IA: Iowa; IC: Intermittent carrier; IEC: Immune evasion cluster; IL: Illinois; IN: Indiana; IQ: Interquartile; MD: Maryland; MI: Michigan; MLST: Multilocus sequence typing; MN: Minnesota; MO: Missouri; MRSA: Methicillin resistant Staphylococcus aureus; MSSA: Methicillin susceptible Staphylococcus aureus; MST: Minimal spanning tree; NC: Non-carrier; NC: North 
Carolina; NE: Nebraska; OH: Ohio; OK: Oklahoma; PA: Pennsylvania; PC: Persistent carrier; PCR: Polymerase chain reaction; S. aureus: Staphylococcus aureus; SD: South Dakota; SNPs: Single nucleotide polymorphisms; spa: Staphylococcus aureus protein A; ST: Sequence type; TPC: True persistent carrier; TX: Texas

\section{Acknowledgements}

The dedicated collaboration of the study participants is greatly appreciated.

\section{Funding}

The study was funded by the National Institute of Occupational Health and Safety via the Upper Midwest Agricultural Safety and Health Center (NIOSH/ CDC - U54OH010170). Whole genome sequencing of selected isolates was funded by the Population Systems Program of the College of Veterinary Medicine, University of Minnesota. Dr. Gustavo Machado provided guidance in the mixed model analysis.

\section{Availability of data and materials}

The datasets generated during and/or analysed during the current study are available from the corresponding author on reasonable request.

\section{Authors' contributions}

Conceived and designed the experiments: PD SS JB. Performed the experiments: JS MY PD. Analyzed the data: JS SS RS TK PD. Wrote the paper: JS MY SS JB RS TK DM PD. All authors read and approved the final manuscript.

\section{Ethics approval and consent to participate}

All procedures were approved by the Institutional Review Board (1111 M06583) and the Institutional Biosafety Committee (1406-31632H) of the University of Minnesota.

\section{Competing interests}

The authors declare that they have no competing interests.

\section{Publisher's Note}

Springer Nature remains neutral with regard to jurisdictional claims in published maps and institutional affiliations.

\section{Author details}

'Department of Veterinary Population Medicine, University of Minnesota, 385 ASVM, 1988 Fitch Ave, St. Paul, MN 55108, USA. ²Department of Veterinary and Biomedical Sciences, University of Minnesota, St. Paul, MN 55108, USA.

Received: 18 January 2017 Accepted: 4 October 2017 Published online: 19 October 2017

\section{References}

1. Voss A, Loeffen F, Bakker J, Klaassen C, Wulf M. Methicillin-resistant Staphylococcus Aureus in pig farming. Emerg Infect Dis. 2005;11(12):1965-6.

2. Chuang YY, Huang YC. Livestock-associated meticillin-resistant Staphylococcus Aureus in Asia: an emerging issue? Int J Antimicrob Agents. 2015;45(4):334-40

3. Khanna T, Friendship R, Dewey C, Weese JS. Methicillin resistant Staphylococcus Aureus colonization in pigs and pig farmers. Vet Microbiol. 2008:128(3-4):298-303.

4. Smith TC, Gebreyes WA, Abley MJ, Harper AL, Forshey BM, Male MJ, Martin HW, Molla BZ, Sreevatsan S, Thakur S, Thiruvengadam M, Davies PR. Methicillin-resistant Staphylococcus Aureus in pigs and farm workers on conventional and antibiotic-free swine farms in the USA. PLoS One. 2013;8(5):e63704.

5. Graveland H, Wagenaar JA, Heesterbeek $H$, Mevius D, van Duijkeren E, Heederik D. Methicillin resistant Staphylococcus Aureus ST398 in veal calf farming: human MRSA carriage related with animal antimicrobial usage and farm hygiene. PLoS One. 2010;5(6):e10990.

6. Van den Eede A, Martens A, Lipinska U, Struelens M, Deplano A, Denis O, Haesebrouck F, Gasthuys F, Hermans K. High occurrence of methicillinresistant Staphylococcus Aureus ST398 in equine nasal samples. Vet Microbiol. 2009:133(1-2):138-44.

7. Nemati M, Hermans K, Lipinska U, Denis O, Deplano A, Struelens M, Devriese LA, Pasmans F, Haesebrouck F. Antimicrobial resistance of old and recent Staphylococcus Aureus isolates from poultry: first detection of livestock-associated methicillin-resistant strain ST398. Antimicrob Agents Chemother. 2008:52(10):3817-9.

8. Sun J, Yang M, Sreevatsan S, Davies PR. Prevalence and characterization of Staphylococcus Aureus in growing pigs in the USA. PLoS One. 2015;10(11):e0143670.

9. Alba P, Feltrin F, Cordaro G, Porrero MC, Kraushaar B, Argudin MA Nykasenoja S, Monaco M, Stegger M, Aarestrup FM, Butaye P, Franco A, Battisti A. Livestock-associated methicillin resistant and methicillin susceptible Staphylococcus Aureus sequence type (CC)1 in European farmed animals: high genetic relatedness of isolates from Italian cattle herds and humans. PLoS One. 2015;10(8):e0137143.

10. Feltrin F, Alba P, Kraushaar B, lanzano A, Argudin MA, Di Matteo P, Porrero MC, Aarestrup FM, Butaye P, Franco A, Battisti A. A livestock-associated, multidrug-resistant, methicillin-resistant Staphylococcus Aureus clonal complex 97 lineage spreading in dairy cattle and pigs in Italy. Appl Environ Microbiol. 2015;82(3):816-21.

11. Sivaraman K, Venkataraman N, Cole AM. Staphylococcus Aureus nasal carriage and its contributing factors. Future Microbiol. 2009;4(8):999-1008.

12. den Heijer CD, van Bijnen EM, Paget WJ, Pringle M, Goossens H, Bruggeman CA, Schellevis FG, Stobberingh EE, Study Team APRES. Prevalence and resistance of commensal Staphylococcus Aureus, including meticillinresistant $S$ aureus, in nine European countries: a cross-sectional study. Lancet Infect Dis. 2013;13(5):409-15.

13. Gorwitz RJ, Kruszon-Moran D, McAllister SK, McQuillan G, McDougal LK, Fosheim GE, Jensen BJ, Killgore G, Tenover FC, Kuehnert MJ. Changes in the prevalence of nasal colonization with Staphylococcus Aureus in the United States, 2001-2004. J Infect Dis. 2008;197(9):1226-34.

14. Muthukrishnan G, Lamers RP, Ellis A, Paramanandam V, Persaud AB, Tafur S, Parkinson CL, Cole AM: Longitudinal genetic analyses of Staphylococcus aureus nasal carriage dynamics in a diverse population. BMC Infect Dis 2013, 13:221-2334-13-221

15. Wertheim HF, Melles DC, Vos MC, van Leeuwen W, van Belkum A, Verbrugh $H A$, Nouwen $J$. The role of nasal carriage in Staphylococcus Aureus infections. Lancet Infect Dis. 2005:5(12):751-62.

16. Sollid JU, Furberg AS, Hanssen AM, Johannessen M. Staphylococcus Aureus: determinants of human carriage. Infect Genet Evol. 2014:21:531-41.

17. Frank DN, Feazel LM, Bessesen MT, Price CS, Janoff EN, Pace NR. The human nasal microbiota and Staphylococcus Aureus carriage. PLoS One. 2010;5(5):e10598.

18. Zipperer A, Konnerth MC, Laux C, Berscheid A, Janek D, Weidenmaier C, Burian M, Schilling NA, Slavetinsky C, Marschal M, Willmann M, Kalbacher $H$, Schittek B, Brotz-Oesterhelt H, Grond S, Peschel A, Krismer B. Human commensals producing a novel antibiotic impair pathogen colonization. Nature. 2016:535(7613):511-6.

19. Guinane CM, Ben Zakour NL, Tormo-Mas MA, Weinert LA, Lowder BV Cartwright RA, Smyth DS, Smyth CJ, Lindsay JA, Gould KA, Witney A, Hinds J, Bollback JP, Rambaut A, Penades JR, Fitzgerald JR. Evolutionary genomics of Staphylococcus Aureus reveals insights into the origin and molecular basis of ruminant host adaptation. Genome Biol Evol. 2010;2:454-66.

20. Lowder BV, Fitzgerald JR. Human origin for avian pathogenic Staphylococcus Aureus. Virulence. 2010;1(4):283-4.

21. Viana D, Comos M, McAdam PR, Ward MJ, Selva L, Guinane CM, GonzalezMunoz BM, Tristan A, Foster SJ, Fitzgerald JR, Penades JR. A single natural nucleotide mutation alters bacterial pathogen host tropism. Nat Genet. 2015;47(4):361-6.

22. Linhares L. A pilot study of the epidemiology of Staphylococcus Aureus in multiple site swine production. Retrieved from the University of Minnesota Digital Conservancy. https://conservancy.umn.edu/handle/11299/162367.

23. van Cleef BA, Verkade EJ, Wulf MW, Buiting AG, Voss A, Huijsdens XW, van Pelt W, Mulders MN, Kluytmans JA. Prevalence of livestock-associated MRSA in communities with high pig-densities in The Netherlands. PLoS One. 2010;5(2):e9385.

24. Verkade $E$, van Benthem B, den Bergh MK, van Cleef B, van Rijen M, Bosch T, Kluytmans J. Dynamics and determinants of Staphylococcus Aureus carriage in livestock veterinarians: a prospective cohort study. Clin Infect Dis. 2013;57(2):e11-7.

25. Cuny C, Nathaus R, Layer F, Strommenger B, Altmann D, Witte W. Nasal colonization of humans with methicillin-resistant Staphylococcus Aureus (MRSA) CC398 with and without exposure to pigs. PLoS One. 2009;4(8):e6800 
26. Bisdorff B, Scholholter JL, Claussen K, Pulz M, Nowak D, Radon K. MRSAST398 in livestock farmers and neighbouring residents in a rural area in Germany. Epidemiol Infect. 2012;140(10):1800-8.

27. Fang HW, Chiang PH, Huang YC. Livestock-associated methicillin-resistant Staphylococcus Aureus ST9 in pigs and related personnel in Taiwan. PLoS One. 2014;9(2):e88826

28. Frana TS, Beahm AR, Hanson BM, Kinyon JM, Layman LL, Karriker LA, Ramirez A, Smith TC. Isolation and characterization of methicillin-resistant Staphylococcus Aureus from pork farms and visiting veterinary students. PLoS One. 2013;8(1):e53738.

29. Armand-Lefevre L, Ruimy R, Andremont A. Clonal comparison of Staphylococcus Aureus isolates from healthy pig farmers, human controls, and pigs. Emerg Infect Dis. 2005;11(5):711-4.

30. Gibbs SG, Green CF, Tarwater PM, Mota LC, Mena KD, Scarpino PV. Isolation of antibiotic-resistant bacteria from the air plume downwind of a swine confined or concentrated animal feeding operation. Environ Health Perspect. 2006;114(7):1032-7.

31. Friese A, Schulz J, Hoehle L, Fetsch A, Tenhagen BA, Hartung J, Roesler U. Occurrence of MRSA in air and housing environment of pig barns. Vet Microbiol. 2012;158(1-2):129-35.

32. van Cleef BA, Graveland $H$, Haenen AP, van de Giessen AW, Heederik D, Wagenaar JA, Kluytmans JA. Persistence of livestock-associated methicillinresistant Staphylococcus Aureus in field workers after short-term occupational exposure to pigs and veal calves. J Clin Microbiol. 2011;49(3):1030-3.

33. van den Broek IV, van Cleef BA, Haenen A, Broens EM, van der Wolf PJ, van den Broek MJ, Huijsdens XW, Kluytmans JA, van de Giessen AW, Tiemersma EW. Methicillin-resistant Staphylococcus Aureus in people living and working in pig farms. Epidemiol Infect. 2009;137(5):700-8.

34. van Cleef BA, van Benthem BH, Verkade EJ, van Rijen MM, Kluytmans-van den Bergh MF, Graveland H, Bosch T, Verstappen KM, Wagenaar JA, Bos ME, Heederik D, Kluytmans JA. Livestock-associated MRSA in household members of pig farmers: transmission and dynamics of carriage, a prospective cohort study. PLoS One. 2015;10(5):e0127190.

35. Linhares LL, Yang M, SreevatsanS, Munoz-Zanzi CA, Torremorell M, Davies PR. The effect of anatomic site and age on detection of Staphylococcus Aureus in pigs. J Vet Diagn Invest 2015;27(1):55-60.

36. Harmsen D, Claus H, Witte W, Rothganger J, Claus H, Turnwald D, Vogel U. Typing of methicillin-resistant Staphylococcus Aureus in a university hospital setting by using novel software for spa repeat determination and database management. J Clin Microbiol. 2003;41(12):5442-8.

37. Enright MC, Day NP, Davies CE, Peacock SJ, Spratt BG. Multilocus sequence typing for characterization of methicillin-resistant and methicillin-susceptible clones of Staphylococcus Aureus. J Clin Microbiol. 2000;38(3):1008-15.

38. Mehrotra M, Wang G, Johnson WM. Multiplex PCR for detection of genes for Staphylococcus Aureus enterotoxins, exfoliative toxins, toxic shock syndrome toxin 1, and methicillin resistance. J Clin Microbiol. 2000;38(3):1032-5.

39. van Cleef BA, van Benthem BH, Verkade EJ, van Rijen M, Kluytmans-van den Bergh MF, Schouls LM, Duim B, Wagenaar JA, Graveland H, Bos ME, Heederik D, Kluytmans JA. Dynamics of methicillin-resistant Staphylococcus Aureus and methicillin-susceptible Staphylococcus Aureus carriage in pig farmers: a prospective cohort study. Clin Microbiol Infect. 2014; 20(10):0764-71.

40. Dierikx CM, Hengeveld PD, Veldman KT, de Haan A, van der Voorde S, Dop PY, Bosch T, van Duijkeren E. Ten years later: still a high prevalence of MRSA in slaughter pigs despite a significant reduction in antimicrobial usage in pigs the Netherlands. J Antimicrob Chemother. 2016;71(9):2414-8.

41. Sun J, Singer RS, Hau SJ, Nicholson TL, Davies PR. Antimicrobial resistance of Staphylococcus Aureus isolated from swine and swine veterinarians in the USA. 2016.

42. Cuny C, Abdelbary M, Layer F, Werner G, Witte W. Prevalence of the immune evasion gene cluster in Staphylococcus Aureus CC398. Vet Microbiol. 2015;177(1-2):219-23.

43. Stegger M, Liu CM, Larsen J, Soldanova K, Aziz M, Contente-Cuomo T, Petersen A, Vandendriessche S, Jimenez JN, Mammina C, van Belkum A, Salmenlinna S, Laurent F, Skov RL, Larsen AR, Andersen PS, Price LB. Rapid differentiation between livestock-associated and livestock-independent Staphylococcus Aureus CC398 clades. PLoS One. 2013;8(11):e79645.

44. Votintseva AA, Miller RR, Fung R, Knox K, Godwin H, Peto TE, Crook DW, Bowden R, Walker AS. Multiple-strain colonization in nasal carriers of Staphylococcus Aureus. J Clin Microbiol. 2014;52(4):1192-200.
45. Goerge T, Lorenz MB, van Alen S, Hubner NO, Becker K, Kock R. MRSA colonization and infection among persons with occupational livestock exposure in Europe: prevalence, preventive options and evidence. Vet Microbiol. 2017;200:6-12.

46. Ritchie SR, Isdale E, Priest P, Rainey PB, Thomas MG. The turnover of strains in intermittent and persistent nasal carriers of Staphylococcus Aureus. J Inf Secur. 2016;72(3):295-301.

47. Williams REO. Healthy carriage of Staphylococcus Aureus: its prevalence and importance. Bacteriol Rev. 1963;27(1):56-71.

48. Verhoeven PO, Gagnaire J, Botelho-Nevers E, Grattard F, Carricajo A, Lucht F, Pozzetto B, Berthelot P. Detection and clinical relevance of Staphylococcus Aureus nasal carriage: an update. Expert Rev Anti-Infect Ther. 2014;12(1):75-89.

49. von Eiff C, Becker K, Machka K, Stammer H, Peters G. Nasal carriage as a source of Staphylococcus Aureus bacteremia. Study group. N Engl J Med. 2001:344(1):11-6.

50. Verhoeven PO, Grattard F, Carricajo A, Lucht F, Cazorla C, Garraud O, Pozzetto B, Berthelot P. An algorithm based on one or two nasal samples is accurate to identify persistent nasal carriers of Staphylococcus Aureus. Clin Microbiol Infect. 2012;18(6):551-7.

51. Ghasemzadeh-Moghaddam H, Neela V, van Wamel W, Hamat RA, Shamsudin MN, Hussin NS, Aziz MN, Haspani MS, Johar A, Thevarajah S, Vos M, van Belkum A. Nasal carriers are more likely to acquire exogenous Staphylococcus aureus strains than non-carriers. Clin Microbiol Infect. 2015;21(11):998.e1-7.

52. Bos ME, Verstappen KM, van Cleef BA, Dohmen W, Dorado-Garcia A, Graveland H, Duim B, Wagenaar JA, Kluytmans JA, Heederik DJ. Transmission through air as a possible route of exposure for MRSA. J Expo Sci Environ Epidemiol. 2016;26(3):263-9.

53. Graveland H, Wagenaar JA, Bergs K, Heesterbeek H, Heederik D. Persistence of livestock associated MRSA CC398 in humans is dependent on intensity of animal contact. PLoS One. 2011;6(2):e16830

54. Bosch T, Verkade E, van Luit M, Landman F, Kluytmans J, Schouls LM. Transmission and persistence of livestock-associated methicillin-resistant Staphylococcus Aureus among veterinarians and their household members. Appl Environ Microbiol. 2015;81(1):124-9.

55. Verkade $E$, Kluytmans-van den Bergh $M$, van Benthem B, van Cleef B, van Rijen M, Bosch T, Schouls L, Kluytmans J. Transmission of methicillinresistant Staphylococcus Aureus CC398 from livestock veterinarians to their household members. PLoS One. 2014;9(7):e100823.

56. Grontvedt CA, Elstrom P, Stegger M, Skov RL, Skytt Andersen P, Larssen KW, Urdahl AM, Angen O, Larsen J, Amdal S, Lotvedt SM, Sunde M, Bjornholt JV. Methicillin-resistant Staphylococcus Aureus CC398 in humans and pigs in Norway: a "one health" perspective on introduction and transmission. Clin Infect Dis. 2016:63(11):1431-8.

57. Nair R, Wu J, Carrel M, O'Brien A, Quick M, Farina S, Wardyn S, Thapaliya D, Grenier D, Smith TC. Prospective multicenter surveillance identifies Staphylococcus Aureus infections caused by livestock-associated strains in an agricultural state. Diagn Microbiol Infect Dis. 2016;85(3):360-366.

58. Larsen J, Petersen A, Sorum M, Stegger M, van Alphen L, Valentiner-Branth $P$, Knudsen LK, Larsen LS, Feingold B, Price LB, Andersen PS, Larsen AR, Skov RL: Meticillin-resistant Staphylococcus aureus CC398 is an increasing cause of disease in people with no livestock contact in Denmark, 1999 to 2011. Euro Surveill 2015, 20(37). doi:10.2807/1560-7917.ES.2015.20.37.30021.

59. Omland O, Hoffmann L. Occupational acquisition of methicillin-resistant Staphylococcus Aureus in humans-a description of MRSA carrier and infected cases from the region of North Jutland in Denmark. Ann Agric Environ Med. 2012;19(4):637-40.

60. VAN Cleef BA, VAN Benthem BH, Verkade EJ, VAN Rijen MM, Kluytmans-VAN DEN Bergh MF, Graveland H, Bosch T, Verstappen KM, Wagenaar JA, Heederik D, Kluytmans JA: Health and health-related quality of life in pig farmers carrying livestock-associated methicillin-resistant Staphylococcus Aureus. Epidemiol Infect 2016, 144(8):1774-1783.

61. Nielsen RT, Kemp M, Holm A, Skov MN, Detlefsen M, Hasman H, Aarestrup FM, Kaas RS, Nielsen JB, Westh H, Kolmos HJ. Fatal septicemia linked to transmission of MRSA clonal complex 398 in hospital and nursing home, Denmark. Emerg Infect Dis. 2016;22(5):900-2. 